\title{
Utilisation de tissus par les adolescentes durant les menstruations à Mangalore, Inde
}

\section{INTRODUCTION}

Durant les menstruations, une hygiène menstruelle adaptée est nécessaire pour éviter des infections et le matériel absorbant utilisé est particulièrement important. ${ }^{1}$ En Inde, une recherche menée dans deux écoles urbaines et rurales à Mangalore rapporte que respectivement 34 et $50 \%$, des adolescentes utilisent des tissus; c'est-à-dire des morceaux de textile, en tant que protection hygiénique durant les menstruations. Un tel usage les expose à des risques accrus d'infection. ${ }^{2}$ L'objectif de cette recherche était d'explorer l'influence des facteurs socioculturels et économiques sur l'utilisation de tissus par les adolescentes à Mangalore.

\section{MÉTHODE}

Nous avons mené une étude qualitative et réalisé 12 entretiens semi-structurés. Les participantes ont été recrutées par nos partenaires académiques du Father Muller College of Nursing (FMCN) en fonction de notre protocole de recherche qui a été approuvé par le comité d'éthique local. L'échantillon de convenance était constitué de 2 gynécologues, 2 infirmières communautaires et 1 pédiatrique, 2 travailleuses sociales, 3 enseignantes et 2 femmes issues de la population générale. Chaque entretien a été retranscrit et analysé séparément puis discuté dans une démarche intersubjective. Une grille d'analyse reprenant les dimensions du modèle transculturel infirmier de Leininger ${ }^{3}$ a permis de dégager les facteurs principaux influençant l'utilisation de tissus. Nos résultats ont été discutés, par entretien téléphonique et en personne, avec deux experts scientifiques indiens. ${ }^{1,2}$

\section{RÉSULTATS}

Les interviewées estimaient que la majorité des adolescentes utilisent des serviettes hygiéniques comme matériel absorbant et une minorité des tissus. La perception de l'utilisation des tissus est divergente: certaines considèrent cette pratique acceptable par rapport à la santé lors d'une hygiène adaptée; d'autres la jugent inadéquate. Les enseignants et professionnels de la santé promeuvent l'utilisation de serviettes hygiéniques tout comme les médias au travers de publicités. Ces derniers sont une source d'information importante. Néanmoins, la transmission du savoir sur les matériaux absorbants se fait essentiellement par les mères, les autres membres féminins de la famille, ainsi que les pairs. Cinq facteurs semblent influencer le choix du matériel absorbant 
(figure 1). L'éducation apparaît comme le facteur majeur menant au choix des serviettes. Malgré les difficultés pratiques de l'utilisation des tissus (lavage, séchage), des ressources financières limitées perpétuent leur utilisation. La culture influence également la perception des menstruations qui demeure taboue, bien que ce sujet soit actuellement plus facilement abordé.

\section{Fig 1}

Facteurs influençant le choix du matériel absorbant pour les adolescentes

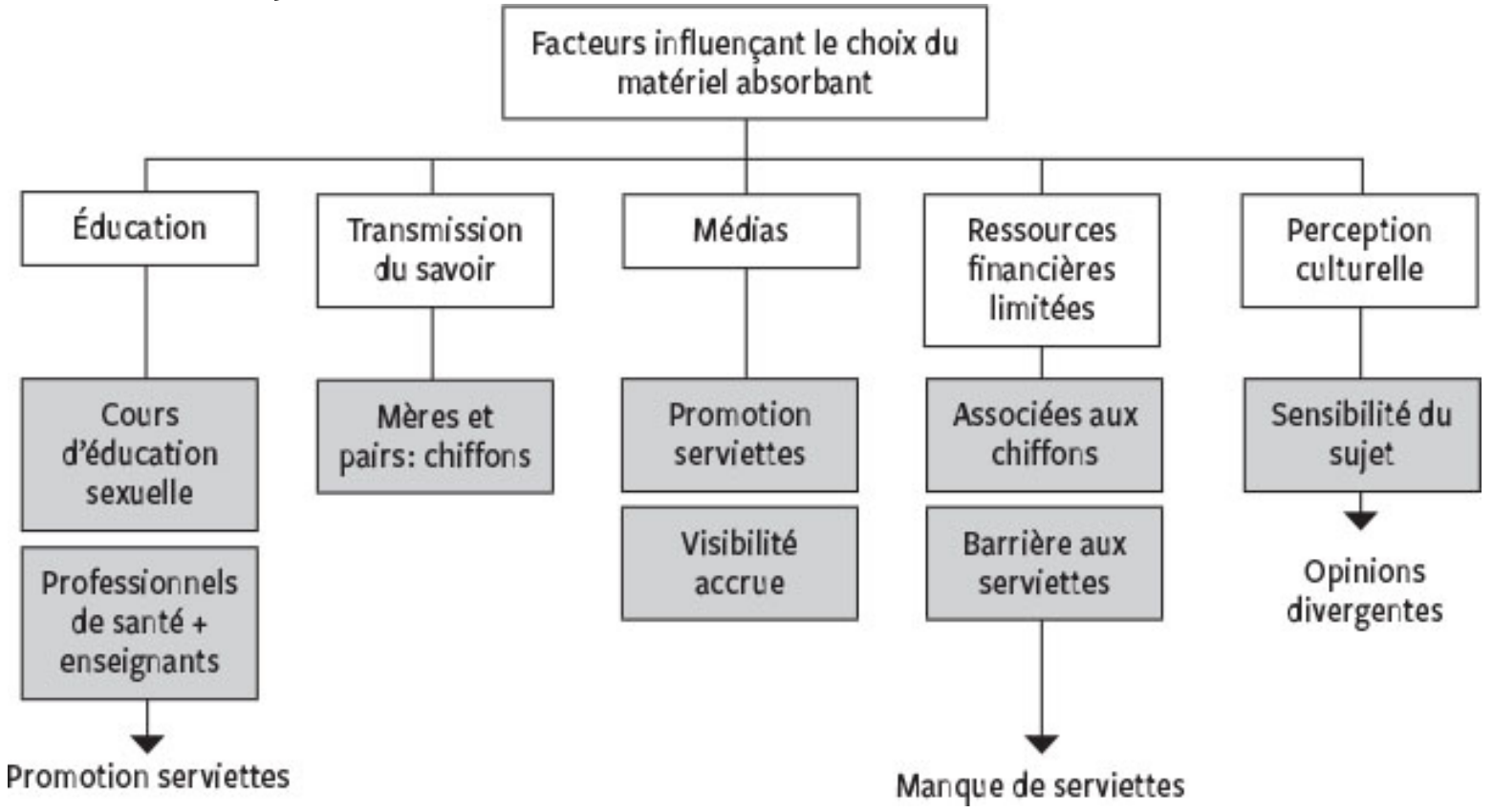

(/var/site/storage/images/rms/2020/rms-n-711/images/rms_711_2008_figo1.jpg/2997860-1-fre-

CH/RMS_711_2008_figo1.jpg_i1140.jpg)

\section{DISCUSSION}

Cette étude, en accord avec la littérature scientifique, ${ }^{1,2}$ montre que les tissus sont encore couramment utilisés. Néanmoins, l'emploi des serviettes hygiéniques croît grâce aux pratiques enseignées actuellement. La scolarité obligatoire et l'impossibilité de laver les tissus à l'école favorisent leur abandon. La visibilité accrue des produits sanitaires dans les médias pourrait également jouer un rôle. Un lien probable existe entre l'usage des tissus, des ressources financières limitées et un bas niveau d'éducation maternel. Aborder la question des menstruations reste un sujet sensible; les professionnels de la santé et les enseignants ont tendance à en parler plus librement, étant exposés à ce sujet. Les interlocutrices rencontrées estimaient qu'au sein de leur contexte catholique, les menstruations ne sont pas taboues. Le milieu socio-éducationnel élevé dont proviennent les participantes, qui est une limite de notre étude, pourrait expliquer qu'elles estiment qu'une majorité de femmes utilisent des serviettes contrairement à ce qu'indique la littérature. ${ }^{1,2}$

\section{Remerciements:}

Nous remercions nos tuteurs.tutrice Dr Séverine Vuilleumier, Pr Blaise Guinchard, et Dr Jacques Gaume; la DGES-VD, l'Université de Lausanne, la Haute École et Institut de Santé La Source, ainsi que le Father Muller College of Nursing.

\section{Bibliographie}


$1 \uparrow$ SM Kotian VK Chaudhary VS Mutya Assessment of knowledge, practice and perception of menstruation among adult women in the reproductive age group, in Mangalore, India. Int J Reprod Contracept Obstet Gynecol 2017 (6)

$2 \uparrow$ P Senapathi H. Kumar A comparative study of menstrual hygiene management among rural and urban adolescent girls in Mangaluru, Karnataka. Int J Community Med Public Health 2018 (5)

\section{$3 \uparrow$ PL. Sagar 2012}

\section{Contact auteur(s)}

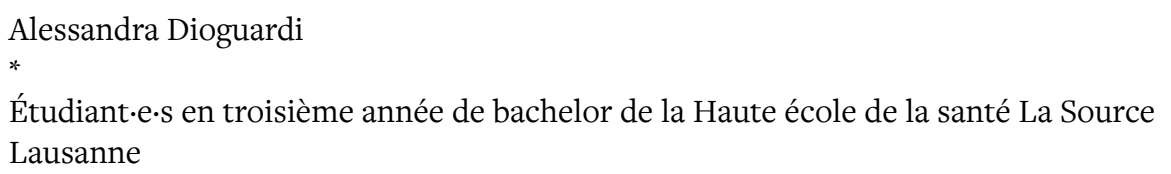

Devina E. Rodrigues

PhD, Father Muller College of Nursing, Mangalore

Inde

Vanessa Gottreux Barron

Étudiant·e.s en troisième année de bachelor de la Haute école de la santé La Source

Lausanne

Elena Orita

$* * * *$

Étudiant.e.s en troisième année de bachelor de la Faculté de biologie et de médecine de l’Université de Lausanne

Sage Zesiger

Étudiant.e.s en troisième année de bachelor de la Faculté de biologie et de médecine de l’Université de Lausanne

2020 (C) Médecine \& Hygiène

Inscrivez-vous à la newsletter

Inscrivez-vous à la newsletter 